Abstracta Iranica Abstracta Iranica

Revue bibliographique pour le domaine irano-aryen

Volume 26 | 2005

Comptes rendus des publications de 2003

\title{
Central Asia. Aspects of Transition. Richmond, Curzon Press, 2003, 288 p., biblio., index.
}

\section{Stéphane A. Dudoignon}

\section{(2) OpenEdition \\ 12 Journals}

\section{Édition électronique}

URL : http://journals.openedition.org/abstractairanica/1211

ISSN : 1961-960X

Éditeur :

CNRS (UMR 7528 Mondes iraniens et indiens), Éditions de l'IFRI

Édition imprimée

Date de publication : 15 mai 2005

ISSN : 0240-8910

\section{Référence électronique}

Stéphane A. Dudoignon, «Central Asia. Aspects of Transition. Richmond, Curzon Press, 2003, 288 p., biblio., index. », Abstracta Iranica [En ligne], Volume 26 | 2005, document 6, mis en ligne le 15 décembre 2005, consulté le 25 septembre 2020. URL : http://journals.openedition.org/abstractairanica/1211

Ce document a été généré automatiquement le 25 septembre 2020

Tous droits réservés 


\title{
Central Asia. Aspects of Transition. Richmond, Curzon Press, 2003, 288 p., biblio., index.
}

\author{
Stéphane A. Dudoignon
}

1 Ce volume d'articles est le produit du séminaire d'études centrasiatiques dirigé à SOAS par Shirin Akiner. Il se subdivise en plusieurs ensembles, portant chacun sur une période de l'histoire de l'Asie Centrale depuis la fin de la Première Guerre mondiale. Une première section est constituée d'études sur la période révolutionnaire et le début de la période soviétique (1917-1924) : après un article de mise au point, sur la base de sources primaires textuelles russes et occidentales, sur le rôle de Mikhaïl Frounze dans l'organisation du Front du Turkestan (Alexander Marshall, « Turkfront: Frunze and the Development of Soviet Counter-Insurgency in Central Asia ", pp. 5-29), on aborde l'impact d'une absence de coordination entre l'émirat de Boukhara et les leaders de l'Autonomie turkestanaise dans l'échec de la résistance vernaculaire à l'emprise bolchévique sur l'Asie Centrale (Paul Bergne, «The Kokand Autonomy, 1917-18: Political Background, Aims and Reasons for Failure», pp. 30-44), puis un article curieusement dépourvu d'apparat critique, sur l'affrontement entre dirigeants communistes principalement ouzbeks et kirghiz au sujet de la délimitation nationale opérée à partir de 1924 (Arslan Koichiev, «Ethno-Territorial Claims in the Ferghana Valley during the Process of National Delimitation, 1924-7», pp. 45-56), avant une contribution sur les réformes agraires des années 1920 en Asie Centrale qui passe en revue quelques ouvrages de souvenirs de représentants de l'émigration turkestanaise en exil et les travaux de chercheurs occidentaux (Gerard o'Neill, «Land and Water 'Reform' in the 1920s: Agrarian Revolution or Social Engineering?», pp. 57-79). Ce premier ensemble est suivi d'un second sur le processus de construction nationale à divers moments de l'histoire du court $20^{\mathrm{e}} \mathrm{s}$. : une première étude comparative passe en revue la littérature scientifique, principalement occidentale, sur la création de la République turque en 1923 et celle de la RSS Ouzbèque en 1924, pour insister sur l'opposition entre la résolution et la continuité dans l'action du régime d'Ankara, d'une part, et de l'autre les nombreuses hésitations, voire l'aporie générale, du régime 
soviétique à Tachkent dans les années 1920-30 face aux enjeux de la construction nationale (Andrew segars, "Nation Building in Turkey and Uzbekistan: The Use of Language and History in the Creation of National Identity ", pp. 80-105); vient ensuite un article sur les problèmes d'identité collective des nationalités exogènes au Kirghizstan (Robert Lowe, "Nation Building and Identity in the Kyrgyz Republic", pp. 106-131), suivi d'une très brève étude sur les utilisations politiques successives de la référence historique au soulèvement du khan Kenesary (de 1837 à 1847) au Kazakhstan après la seconde guerre mondiale (Henri Fruchet, "The Use of History: The Soviet Historiography of Khan Kenesary Kasimov », pp. 132-145). Un dernier ensemble d'articles porte sur les transformations économiques et sociales de l'Asie Centrale postsoviétique. Le premier esquisse, selon un schéma assez classique, des pistes de comparaison entre le développement économique et social de l'Asie Centrale soviétique dans les années 1950-90 et celui que l'on observe pendant la même période à la fois dans les régions européennes de l'URSS et les pays du Proche-Orient (Alex stringer, "Soviet Development in Central Asia », pp.146-166). Il est suivi par une étude qui insiste sur l'instrumentation politique des questions d'écologie par les pouvoirs présidentiels issus de la période soviétique, et sur les faibles perspectives de participation politique populaire dans ces questions (Lars Jalling, « Environment Issues in Central Asia: A Source of Hope or Despair?», pp. 167-179). Vient ensuite une étude comparative assez superficielle, basée pour l'essentiel sur des articles de presse, sur les progrès de l'islam politique radical au Kazakhstan et en Ouzbékistan (Tom EverettHeath, «Instability and Identity in a Post-Soviet World : Kazakhstan and Uzbekistan ", pp. 181-204). Elle est suivie d'une contribution beaucoup plus substantielle sur la politisation des unités de voisinage en Ouzbékistan depuis les années 1990 (Elise Massicard \& Tommaso Trevisani, "The Uzbek Mahalla: Between State and Society", pp. 205-218). Le volume se conclut par deux études panoramiques, l'une sur les progrès $\mathrm{du}$ «fondamentalisme » en Asie Centrale, laquelle se réduit à un commentaire de la bibliographie accessible en anglais (Petra steinberger, "'Fundamentalism' in Central Asia: Reasons, Reality and Prospects", pp. 219-243), l'autre sur les ressources hydrauliques comme enjeu économique et politique majeur des années à venir en Asie Centrale (Karl wegerich, « Water: The Difficult Path to a Sustainable Future for Central Asia », pp. 244-263). L'ensemble laisse une impression générale de travail de synthèse fondé, pour l'essentiel, sur la bibliographie anglo-saxonne existante. Des bonnes études relevant d'un travail de second cycle voisinent avec un nombre plus réduit d'articles plus achevés, témoignant d'un important travail de terrain, les unes comme les autres étant réunies par un même goût de la théorisation. 
INDEX

Thèmes : 1.2. Recueils d'articles : revues, colloques, ouvrages collectifs

Mots-clés : réforme agraire, fondamentalisme, economie, ecologie

Keywords : land reform, economy, ecology, fundamentalism, Central Asia

nompropre Mikhaïl Frounze, Kokand, Ouzbékistan, Kirghizstan, Turquie, Ferghana, Kazakhstan, Boukhara, Asie Centrale, Bukara, Front du Turkestan, Turkestan Front

\section{AUTEURS}

\section{STÉPHANE A. DUDOIGNON}

Université Marc Bloch - Strasbourg 